\title{
SeqAssist: a novel toolkit for preliminary analysis of next-generation sequencing data
}

\author{
Yan Peng ${ }^{1}$, Andrew S Maxwell ${ }^{1}$, Natalie D Barker ${ }^{2}$, Jennifer G Laird ${ }^{3}$, Alan J Kennedy ${ }^{3}$, Nan Wang ${ }^{1}$, \\ Chaoyang Zhang $^{1^{*}}$, Ping Gong ${ }^{2^{*}}$ \\ From 11th Annual MCBIOS Conference \\ Stillwater, OK, USA. 6-8 March 2014
}

\begin{abstract}
Background: While next-generation sequencing (NGS) technologies are rapidly advancing, an area that lags behind is the development of efficient and user-friendly tools for preliminary analysis of massive NGS data. As an effort to fill this gap to keep up with the fast pace of technological advancement and to accelerate data-to-results turnaround, we developed a novel software package named SeqAssist ("Sequencing Assistant" or SA).

Results: SeqAssist takes NGS-generated FASTQ files as the input, employs the BWA-MEM aligner for sequence alignment, and aims to provide a quick overview and basic statistics of NGS data. It consists of three separate workflows: (1) the SA_RunStats workflow generates basic statistics about an NGS dataset, including numbers of raw, cleaned, redundant and unique reads, redundancy rate, and a list of unique sequences with length and read count; (2) the SA_Run2Ref workflow estimates the breadth, depth and evenness of genome-wide coverage of the NGS dataset at a nucleotide resolution; and (3) the SA_Run2Run workflow compares two NGS datasets to determine the redundancy (overlapping rate) between the two NGS runs. Statistics produced by SeqAssist or derived from SeqAssist output files are designed to inform the user: whether, what percentage, how many times and how evenly a genomic locus (i.e., gene, scaffold, chromosome or genome) is covered by sequencing reads, how redundant the sequencing reads are in a single run or between two runs. These statistics can guide the user in evaluating the quality of a DNA library prepared for RNA-Seq or genome (re-)sequencing and in deciding the number of sequencing runs required for the library. We have tested SeqAssist using a synthetic dataset and demonstrated its main features using multiple NGS datasets generated from genome re-sequencing experiments.
\end{abstract}

Conclusions: SeqAssist is a useful and informative tool that can serve as a valuable "assistant" to a broad range of investigators who conduct genome re-sequencing, RNA-Seq, or de novo genome sequencing and assembly experiments.

\section{Background}

High throughput next-generation sequencing (NGS) technologies are capable of generating massive amounts of data in the form of paired-end or single-end reads with either fixed or variable lengths. The size of data files is often in the magnitude of mega- or giga-bytes (up to 1000 giga base pairs or $\mathrm{Gb}$ in a single sequencing run)

\footnotetext{
*Correspondence: Chaoyang.Zhang@usm.edu; Ping.Gong@usace.army.mil ${ }^{1}$ School of Computing, University of Southern Mississippi, Hattiesburg, MS 39406, USA

${ }^{2}$ Badger Technical Services, LLC, San Antonio, TX 78216, USA

Full list of author information is available at the end of the article
}

and is likely to increase further in the years to come. While sequencing costs have dropped precipitously and sequencing speed and efficiency have risen exponentially, development of computational tools for preliminary analysis of these gigantic datasets have lagged behind data generation. Hence, there is an increasing demand for efficient and user-friendly programs for preliminary sequencing data analysis.

Currently, there are four commercially predominant NGS platforms, including Illumina/Solexa, Roche/454, $\mathrm{ABI} / \mathrm{SOLiD}$ and $\mathrm{ABI} / \mathrm{Ion}$ Torrent $[1,2]$. These massively parallel DNA sequencing technologies have been applied 
to transcriptome sequencing (RNA-Seq), de novo genome sequencing, and genome re-sequencing. RNA-Seq is a widely used approach to transcriptomic profiling $[3,4]$. Two representative efforts in de novo genome sequencing are the Genome $10 \mathrm{~K}$ project to obtain whole genome sequences for 10,000 vertebrate species [5-7] and the $5 \mathrm{~K}$ Insect Genome Initiative (i5K) to sequence the genomes of 5,000 arthropod species [8,9]. Genome re-sequencing is an experimental procedure that involves sequencing individual organisms whose genome is already known [10]. As a new genomics approach, genome re-sequencing has been applied to a wide range of fundamental and applied biological research including genetics, evolution, biomedicine, human diseases and environmental health, with good examples being the 1000 Genomes Project [11] and the Cancer Genomes project [12].

Prior to in-depth analysis of NGS deep sequencing data, e.g., differential gene expression and alternative splicing analysis for RNA-Seq studies, structural variants identification for genome re-sequencing studies, and genome assembly for de novo genome sequencing studies, investigators are often concerned about the following issues: (1) basic statistics of a sequencing run such as total numbers of raw, cleaned, and unique reads as well as the degree of reads redundancy; (2) sequencing library quality, i.e., does the library truly represent the genome of the re-sequenced organism, and (3) the number of sequencing runs required, i.e., how many runs are necessary to get a full representation of the sequencing library or to suffice a de novo genome assembly. Although many tools such as Partek Genomics Suite, CLC Genomic Workbench, or noncommercial platforms like Galaxy and GenePattern are currently available and capable of indirectly addressing these issues, one would have to possess some basic bioinformatics training and script writing skills in order to manipulate and turn the generated results into useful and straightforward information that can be easily understood by an experimentalists. Motivated by filling this gap, the limitations of existing tools, and also driven by the demand for accelerating data-toresults turnaround, we have developed a novel toolkit named SeqAssist ("Sequencing Assistant", acronym: SA). SeqAssist specifically addresses the aforementioned three issues and provides investigators who conduct RNA-Seq, de novo genome sequencing or genome re-sequencing experiments with a quick overview and preliminary analysis of their NGS data.

\section{Implementation}

SeqAssist was programmed using Perl with an additional Java-enabled Graphic User Interface to enhance efficiency and user-friendliness. It currently consists of three separate workflows: SA_RunStats, SA_Run2Ref and SA_Run2Run. SA_RunStats generates the basic statistics such as total number of raw and cleaned reads, length and copy number of unique sequences, and reads redundancy in a single sequencing run or a pooled dataset of several runs (see Figure 1a). The input of this workflow is a FASTQ-formatted sequencing data file. The data file is preprocessed to trim off adaptors and low quality read ends with a default cutoff of base-calling quality score (Q) at 20, followed by removal of $\mathrm{N}$-containing reads. Then, the cleaned reads are aligned against each other using BWA-MEM (acronym for Burrows-Wheeler Aligner-Maximal Exact Match), one of the three Burrows-Wheeler Transform-based algorithms in the BWA software package $[13,14]$. BWA-MEM is a robust, fast and accurate aligner that supports paired-end reads, performs chimeric alignment, and tolerates sequencing errors (http://arxiv.org/abs/1303.3997v2). Based on the alignment information in the BWA-MEM-generated SAM (acronym for Sequence Alignment/Map format) file [15], the number of unique reads is counted and both identical and inclusive (i.e., redundant) reads are removed. Two reads are considered identical if they match 100\% with each other and they are of equal length, while inclusive reads are defined as the sub-sequences of a longer read and only the longest read is kept as the unique read. The redundancy rate is calculated as the percentage of redundant reads in the total number of unique cleaned reads [see $E q$. (1) for formula]. The output of this workflow includes the total numbers of raw, cleaned, redundant and unique reads, and the redundancy rate. Also included in the output is a tab-delimited plain text file that lists all unique sequences along with their length and read count (copy number). This file can be used to further infer gene expression levels if the run data is produced for an RNA-Seq experiment.

$$
\text { Redundancy rate }(\%)=\frac{\text { number of redundant reads }}{\text { total number of unique cleaned reads }} \times 100 \%
$$

SA_Run2Ref analyzes the breadth, depth and evenness of genome-wide coverage of an individual or pooled sequencing dataset at a nucleotide resolution. Coverage breadth is defined as the percentage of a reference sequence (i.e., gene, scaffold/chromosome, or entire genome) that is covered by sequencing reads [Eq. (2)]; coverage depth is defined as the average times a reference sequence is covered [Eq. (3)]; and coverage evenness is defined as the coefficient of variance of scaffold coverage breadth $[E q$. (4)]. Therefore, outputs from SA_Run2Ref can inform what genomic loci are covered and how a genomic locus (gene), scaffold or the entire genome is covered. In the SA_Run2Ref workflow (Figure 1b), cleaned reads are aligned against the reference genome sequence, generating a SAM file. Information stored in columns 3, 4 and 6 for each alignment in the SAM file represents mandatory fields RNAME (reference sequence 


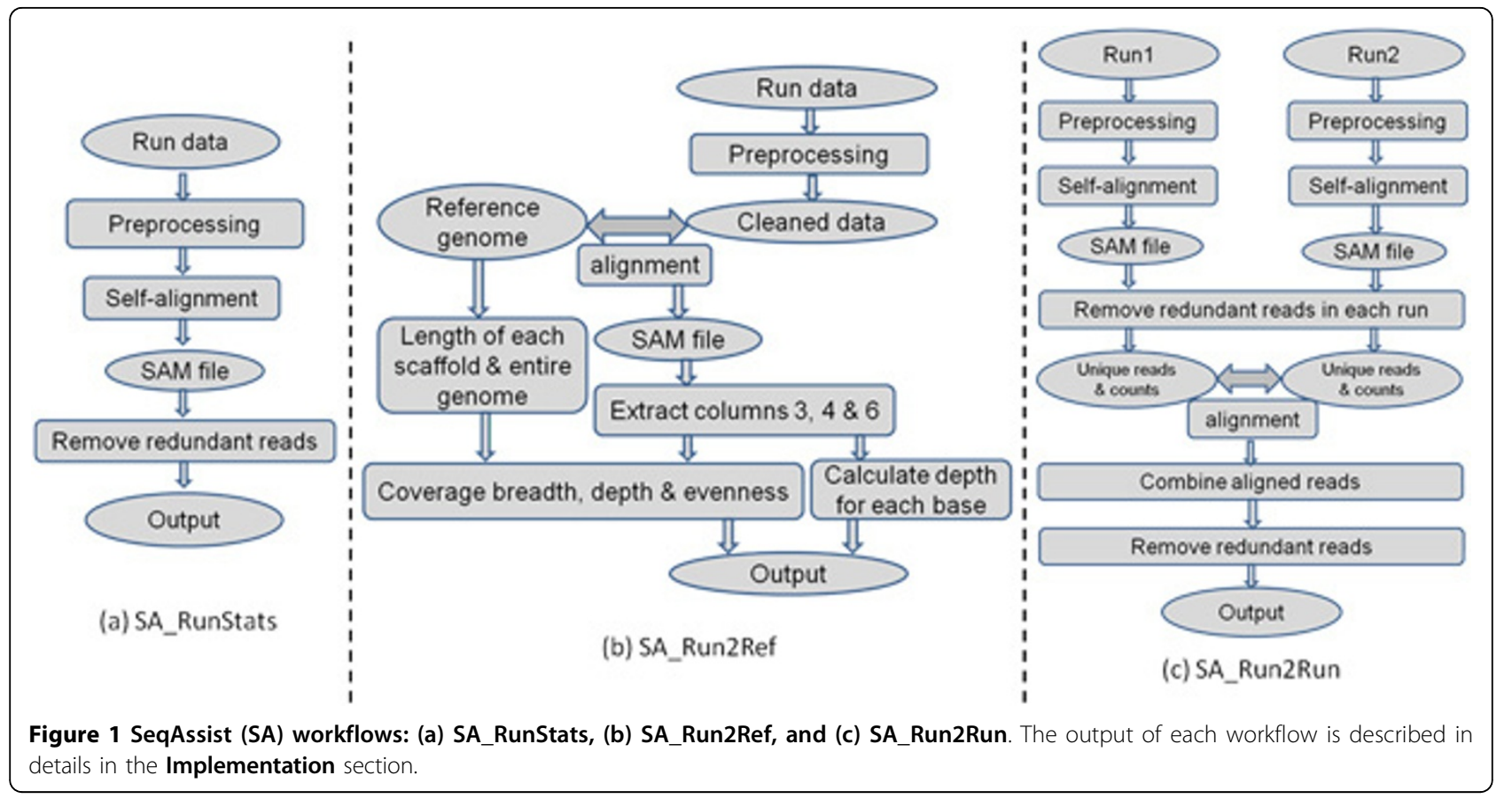

name), POS (1-based leftmost mapping position), and CIGAR (CIGAR string), respectively [15]. This information is extracted along with the length of each scaffold of reference genome to compute scaffold coverage breadth and depth and genome coverage evenness. These statistics are provided in the output files. The output also includes a plain-text file that records the coverage depth of each individual base on the entire genome. This file can be used as an input for genome browser tools to visualize coverage depth of any genomic regions. In case that the user conducts an RNA-Seq experiment and provides gene model sequences (instead of scaffold or chromosome sequences) as the input, the workflow will calculate coverage breadth and depth for each gene model. This information can be readily transformed into gene expression measurements.

$$
\begin{aligned}
& \text { Coverage breadth }(\%)=\frac{\text { number of reference bases mapped by sequencing reads }}{\text { length of the reference sequence in bases }} \times 100 \% \\
& \text { Coverage depth }=\frac{\text { total number of bases mapped to the reference }}{\text { length of the reference sequence in bases }} \\
& \text { Coverage evenness }=\frac{\text { standard deviation of scaffold coverage breadth }}{\text { average scaffold coverage breadth }}
\end{aligned}
$$

SA_Run2Run compares two separate sequencing datasets generated for the same or different DNA libraries, computes the basic statistics for each individual dataset, and estimates the redundancy rate between the two datasets. SA_Run2Run informs the user of the redundancy level both within each individual run and between two sequencing runs. Like the SA_RunStats workflow (Figure 1a), each input run dataset in the SA_Run2Run workflow (Figure 1c) independently goes through the same preprocessing, self-alignment and removing redundant reads steps to generate two new datasets containing unique cleaned sequences. Then, the two new datasets are aligned against each other using BWA-MEM, generating a new SAM file. After combining all aligned reads, reads common to both datasets (i.e., overlapping reads) are identified, the counts of redundant reads (identical or inclusive) are calculated for both overlapping and nonoverlapping reads. The output statistics from SA_Run2Run include total numbers of raw reads, cleaned reads, and unique reads (after removing identical reads and inclusive reads), and numbers of total and unique overlapping reads. The redundancy rates within each dataset and between the two datasets can be further derived from these statistics. Similar to the SA_RunStats output, a list of unique sequences along with their length and count number is provided for each run. However, different from the SA_RunStats output, the list generated by SA_Run2Run is broken into two files, one containing overlapping reads and the other non-overlapping reads. To compare two paired-end sequencing runs, one has to run this workflow twice: Run1_R1.fastq vs. Run2_R1. fastq and Run1_R2.fastq vs. Run2_R2.fastq. The SA_Run2Run workflow intends to guide the user in deciding whether to perform more runs on a sequencing library by looking at the percentage of reads in a new run covered by the reads in a previous run or the pooled reads of multiple previous runs. 


\section{Results and discussion}

To test all SeqAssist workflows, a synthetic dataset was generated by (1) clipping 10 distinct fragments with a length of $150 \mathrm{bp}$ at different loci of the Escherichia coli str. K-12 substr. MG1655l genome (NCBI Reference Sequence Accession No. NC_000913.3, available at http://www.ncbi. nlm.nih.gov/nuccore/556503834? report=fasta) to construct 10 artificial chromosomes, (2) clipping 10 sequences of 75-100 bp in length from each artificial chromosome, and (3) repeating each sequence 10 times. These steps resulted in a dataset of 1,000 reads and a reference genome consisting of 10 short artificial chromosomes, both of which were used to test the SA_RunStats and SA_Run2Ref workflows. The synthetic dataset was further split in two halves to create Run 1 and Run 2 that were used to test the SA_Run2Run workflow. SeqAssist output results for the synthetic dataset are in agreement with expected results (see files in the SeqAssist software package for details), validating the scripts coded for all three workflows.

Here we demonstrate the applications of SeqAssist to preliminary analysis of multiple experimental NGS datasets. As the SA_RunStats workflow is an integral part of the SA_Run2Run workflow, we focus on the SA_Run2Ref and SA_Run2Run workflows in the following experiments. All the experiments were performed on a Dell M710 Blade server equipped with $283 \mathrm{~GB}$ of DDR3 memory at 1,066 $\mathrm{MHz}$ speed, an Intel ${ }^{\circledR}$ Xeon ${ }^{\circledR}$ E5630 CPU Quad-core that runs at $2.53 \mathrm{GHz}$, and two separate hard drives of $1.3 \mathrm{~TB}$ and 2.9 TB. The operating system was Red Hat Enterprise Linux Server release 6.3 (Santiago) using the CentOS 64bit distribution.

\section{Experimental NGS datasets}

We selected multiple NGS datasets and two organisms of contrasting genomic complexity to demonstrate the features of SeqAssist. These datasets represented both fixed and variable length reads generated on Illumina and 454 sequencing platforms, respectively. The chosen organisms were the bacteria Escherichia coli, a prokaryote with a simple and small circular genome of 4.6 Mb in length [16], and the water flea Daphnia pulex, an eukaryote with a recently published draft genome consisting of 5,191 scaffolds with a total length of ca. $200 \mathrm{Mb}$ [17].

Two datasets obtained from an E. coli genome sequencing project were downloaded from http://data.clovr.org/. One dataset (named Ecoli_454_500K) contains 500,000 shotgun 454 titanium sequences (variable length reads in SFF format), and the other (named Ecoli_I4M_R1 and Ecoli_I4M_R2) contains 4,000,000 paired-end shotgun Illumina sequences $(2 \times 49$-bp fixed length reads in FASTQ format). The Bio.SeqIO module in Biopython (http://biopython.org/wiki/Main_Page) was employed to convert the SFF format to the FASTQ format for the 454 dataset by the command \$ python -c "from Bio import SeqIO; SeqIO.convert('in.sff, 'sff', 'out.fastq', 'fastq');". The $D$. pulex datasets were collected in-house by repeatedly sequencing two libraries, each of which was prepared from genomic DNA isolated from an individual animal. One animal came from a population named ECT (acronym for "Environmental Consulting \& Testing", the vendor from which it was obtained, Superior, WI, USA) and the other from another population named TCO (acronym for "The Chosen One", kindly donated by Dr. Norman D. Yan, York University, Toronto, ON, Canada).

To demonstrate the scalability of SeqAssist, we have also chosen a human genome re-sequencing dataset of the CEU HapMap individual NA12878 at a 15 -fold coverage with an insert size of $300 \mathrm{bp}$ and $3.6 \%$ duplicate reads. The dataset (SRA ID: ERX012406) consists of 7 paired-end Illumina Genome Analyzer IIx runs and is downloadable from NCBI's SRA database at http://www. ncbi.nlm.nih.gov/sra/?term=ERX012406. The reference diploid human genome (hg18) consists of 23 pairs of chromosomes or 3,234 Mb in total.

\section{The SA_Run2Ref workflow}

The ECT D. pulex gDNA library was sequenced twice without multiplexing, generating two paired-end sequencing datasets, ECT and ECT_rerun. These two datasets as well as their combined dataset were run through the SA_Run2Ref workflow, producing statistics presented in Table 1 . Approximately $88 \%$ of the cleaned reads from the ECT or the ECT_rerun dataset was mapped to the reference genome, covering $76 \%$ of the 5,191 scaffolds or $64 \%$ of the entire genome at a 9-fold depth. The combined dataset covered less than $1 \%$ more scaffolds than individual datasets, and it also had similar genome coverage breadth and evenness as the two separate datasets, even though it doubled the genome coverage depth. The distribution of scaffold coverage breadth showed a very similar pattern with ca. 1200 scaffolds uncovered for all three datasets (Figure 2). In comparison with the two separate datasets, the combined dataset covered 830 and 895 more scaffolds at $>4$-fold depth or 700 and 774 more at $>10$ fold depth than the ECT and the ECT_rerun datasets, respectively (Figure 2). The number of scaffolds with a coverage breadth of $50 \%$ or less in the two separate datasets was 188 (ECT) or 218 (ECT_rerun) more than that in the combined dataset. These results indicate that the additional sequencing run (ECT_rerun) did not improve much coverage breadth or evenness, and that the two runs covered almost the same scaffolds.

The TCO D. pulex library was split into two fractions: a large fraction $(\mathrm{LF}$, insert size $=572 \mathrm{bp}$ ) and a small fraction (SF, $269 \mathrm{bp}$ ). Each fraction was sequenced five times along with 35 other indexed libraries in a multiplexing fashion using Illumina MiSeq, except for the 
Table 1 Basic statistics produced by SA_Run2Ref for two sequencing run datasets

\begin{tabular}{|c|c|c|c|}
\hline Illumina MiSeq runs (read length $=2 \times 151 \mathrm{bp}$ ) & ECT & ECT_rerun & $\mathrm{ECT}+\mathrm{ECT}$ rerun \\
\hline Total number of raw paired-end reads & $7,575,822$ & $7,064,035$ & $14,639,857$ \\
\hline Total number of cleaned reads & $7,524,261$ & $7,041,454$ & $14,565,715$ \\
\hline Total number of reads mapped to reference genome & $6,573,572$ & $6,193,164$ & $12,766,736$ \\
\hline Mapped/Cleaned reads (\%) & 87.37 & 87.95 & 87.65 \\
\hline Total number of scaffolds in reference genome & 5,191 & 5,191 & 5,191 \\
\hline Number of covered reference scaffolds & 3,960 & 3,948 & 3,998 \\
\hline Covered/Total scaffolds (\%) & 76.29 & 76.05 & 77.02 \\
\hline Genome coverage breadth (\%) & 64.48 & 64.32 & 66.12 \\
\hline Genome coverage depth & 9.24 & 8.67 & 17.91 \\
\hline standard deviation of scaffold coverage depth & 96.11 & 91.88 & 186.95 \\
\hline average scaffold coverage depth & 16.27 & 15.41 & 31.33 \\
\hline Genome coverage evenness & 6.79 & 6.86 & 6.82 \\
\hline Run time (min) & 44.6 & 42.0 & 81.9 \\
\hline
\end{tabular}

The two datasets of paired-end reads were generated using Illumina MiSeq by sequencing the same genomic DNA (gDNA) library prepared for a water flea (Daphnia pulex) from an ECT population. Library preparation involved shearing of extracted gDNA using a Covaris M220 focused-ultrasonicator (Woburn, MA). The average of library insert size distribution was $301 \mathrm{bp}$.

fifth run of LF (LF5) which was pooled with 5 other indexed libraries (Table 2). Hence, the quantity of reads in each LF or SF dataset is equivalent to $1 / 36$ (or $1 / 6$ for LF5) of a MiSeq run. As more datasets were pooled to form new reads collections as input to SA_Run2Ref, the ratio of mapped to cleaned reads remained stable at $82 \%$ to $85 \%$ (Table 2), and the scaffold coverage evenness had little change (Figure 3). Although the genome coverage depth steadily increased as more runs were added to the reads collection, the genome coverage breadth increased simultaneously until LF5 was added and then reached a plateau (Figure 3). The addition of 2.2 million SF reads raised coverage breadth by only $3 \%$ (Table 2 and Figure 3). The change in the distributions

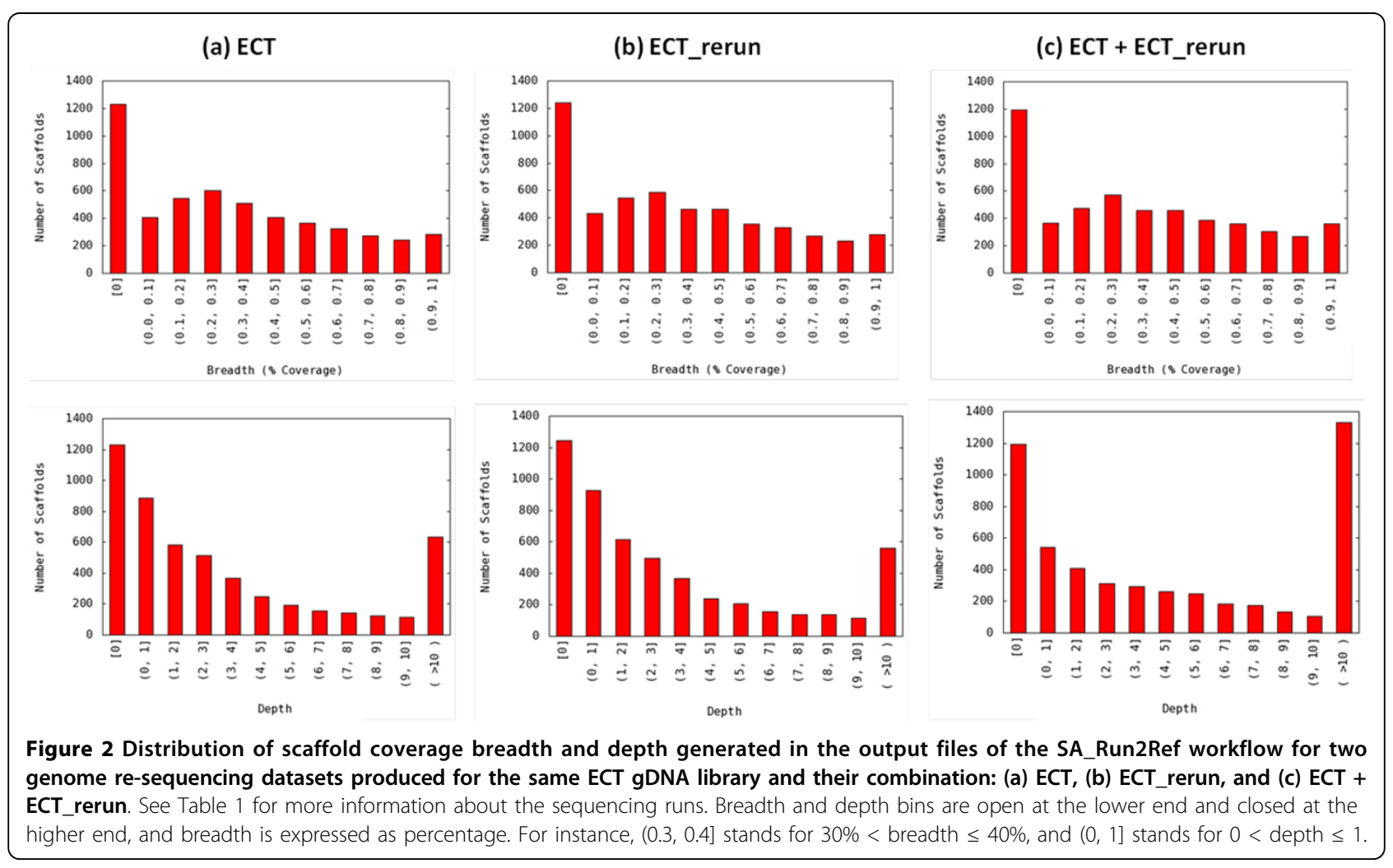


Table 2 Sequencing datasets and genome mapping of the Daphnia pulex TCO library

\begin{tabular}{|c|c|c|c|c|c|c|c|c|}
\hline $\begin{array}{l}\text { Reads } \\
\text { collection }\end{array}$ & Sequencing runs/collection & $\begin{array}{l}\text { Library } \\
\text { fraction }\end{array}$ & $\begin{array}{l}\text { Raw } \\
\text { reads }\end{array}$ & $\begin{array}{l}\text { Cleaned } \\
\text { reads }\end{array}$ & $\begin{array}{l}\text { Mapped } \\
\text { reads }\end{array}$ & $\begin{array}{l}\text { Mapped/Cleaned } \\
\text { reads (\%) }\end{array}$ & $\begin{array}{c}\text { Run time } \\
(\mathrm{min})\end{array}$ & $\begin{array}{c}\text { Added run (multiplex, } \\
\text { read length) }\end{array}$ \\
\hline LF1 & LF1 & Large only & 383,575 & 381,612 & 311,919 & 81.74 & 7.1 & LF1 $(36 \times, 2 \times 151)$ \\
\hline LF1-2 & $L F 1+L F 2$ & Large only & $1,083,738$ & $1,076,671$ & 907,601 & 84.30 & 13.8 & $\operatorname{LF} 2(36 \times, 2 \times 251)$ \\
\hline LF1-3 & $\mathrm{LF} 1+\mathrm{LF} 2+\mathrm{LF} 3$ & Large only & $1,782,006$ & $1,743,523$ & $1,478,140$ & 84.78 & 21.7 & LF3 $(36 \times, 2 \times 251)$ \\
\hline LF1-4 & $\mathrm{LF} 1+\mathrm{LF} 2+\mathrm{LF} 3+\mathrm{LF} 4$ & Large only & $2,218,000$ & $2,177,265$ & $1,848,979$ & 84.92 & 26.1 & LF4 $(36 \times, 2 \times 251)$ \\
\hline LF1-5 & LF1+LF2+LF3+LF4+LF5 & Large only & $4,242,048$ & $4,178,856$ & $3,524,528$ & 84.34 & 45.9 & $\operatorname{LF5}(6 \times, 2 \times 251)$ \\
\hline LF1-5SF1 & LF1+LF2+LF3+LF4+LF5+SF1 & $\begin{array}{l}\text { Large }+ \\
\text { Small }\end{array}$ & $4,542,917$ & $4,478,675$ & $3,766,787$ & 84.10 & 48.1 & SF1 $(36 \times, 2 \times 151)$ \\
\hline $\begin{array}{l}\text { LF1-5 SF1- } \\
2\end{array}$ & $\begin{array}{c}\mathrm{LF} 1+\mathrm{LF} 2+\mathrm{LF} 3+\mathrm{LF} 4+\mathrm{LF} 5+\mathrm{SF} 1 \\
+\mathrm{SF} 2\end{array}$ & $\begin{array}{l}\text { Large }+ \\
\text { Small }\end{array}$ & $5,084,493$ & $5,014,933$ & $4,204,692$ & 83.84 & 50.6 & SF2 $(36 \times, 2 \times 151)$ \\
\hline $\begin{array}{c}\text { LF1-5 SF1- } \\
3\end{array}$ & $\begin{array}{c}\mathrm{LF} 1+\mathrm{LF} 2+\mathrm{LF} 3+\mathrm{LF} 4+\mathrm{LF} 5+\mathrm{SF} 1 \\
+\mathrm{SF} 2+\mathrm{SF} 3\end{array}$ & $\begin{array}{l}\text { Large + } \\
\text { Small }\end{array}$ & $5,530,560$ & $5,457,878$ & $4,561,648$ & 83.58 & 52.7 & SF3 $(36 \times, 2 \times 151)$ \\
\hline $\begin{array}{c}\text { LF1-5 SF1- } \\
4\end{array}$ & $\begin{array}{c}\mathrm{LF} 1+\mathrm{LF} 2+\mathrm{LF} 3+\mathrm{LF} 4+\mathrm{LF} 5+\mathrm{SF} 1 \\
+\mathrm{SF} 2+\mathrm{SF} 3+\mathrm{SF} 4\end{array}$ & $\begin{array}{l}\text { Large }+ \\
\text { Small }\end{array}$ & $5,920,185$ & $5,845,827$ & $4,872,885$ & 83.36 & 54.8 & SF4 $(36 \times, 2 \times 151)$ \\
\hline $\begin{array}{l}\text { LF1-5 SF1- } \\
5\end{array}$ & $\begin{array}{c}\mathrm{LF} 1+\mathrm{LF} 2+\mathrm{LF} 3+\mathrm{LF} 4+\mathrm{LF} 5+\mathrm{SF} 1 \\
+\mathrm{SF} 2+\mathrm{SF} 3+\mathrm{SF} 4+\mathrm{SF} 5\end{array}$ & $\begin{array}{l}\text { Large }+ \\
\text { Small }\end{array}$ & $6,411,123$ & $6,333,054$ & $5,270,616$ & 83.22 & 56.5 & SF5 $(36 \times, 2 \times 151)$ \\
\hline
\end{tabular}

All the NGS run datasets were generated by sequencing the TCO gDNA library which was split into two fractions: a large fraction (LF) with an average insert size of $572 \mathrm{bp}$ and a small fraction (SF) with an average insert size of $269 \mathrm{bp}$. An Illumina MiSeq was used for sequencing and both fractions were each sequenced five times in a $36 \times$ or $6 \times$ multiplexing fashion, resulting in datasets LF1 to LF5 and SF1 to SF5. The reads collections were mapped to a D. pulex reference genome by running the SA_Run2Ref workflow.

of scaffold coverage depth and breadth also supports this conclusion. Except the bin for non-covered scaffolds, the number of scaffolds in every bin increased continuously for both coverage breadth and depth from collection LF1 (Figure 4a) to LF1-5 (Figure 4b), but little difference was observed in the scaffold numbers for coverage breadth between LF1-5 and LF1-5SF1-5 collections (Figure 4c).

\section{The SA Run2Run workflow}

The R1 and R2 files of paired-end sequencing datasets were run separately through the SA_Run2Run workflow. The E. coli datasets (Ecoli_454_500K, Ecoli_I4M_R1 and Ecoli_I4M_R2) were split into two halves to create two runs in order to run this workflow (Table 3). For resequencing of the $E$. coli genome, output statistics suggest that the paired-end Illumina dataset has a higher

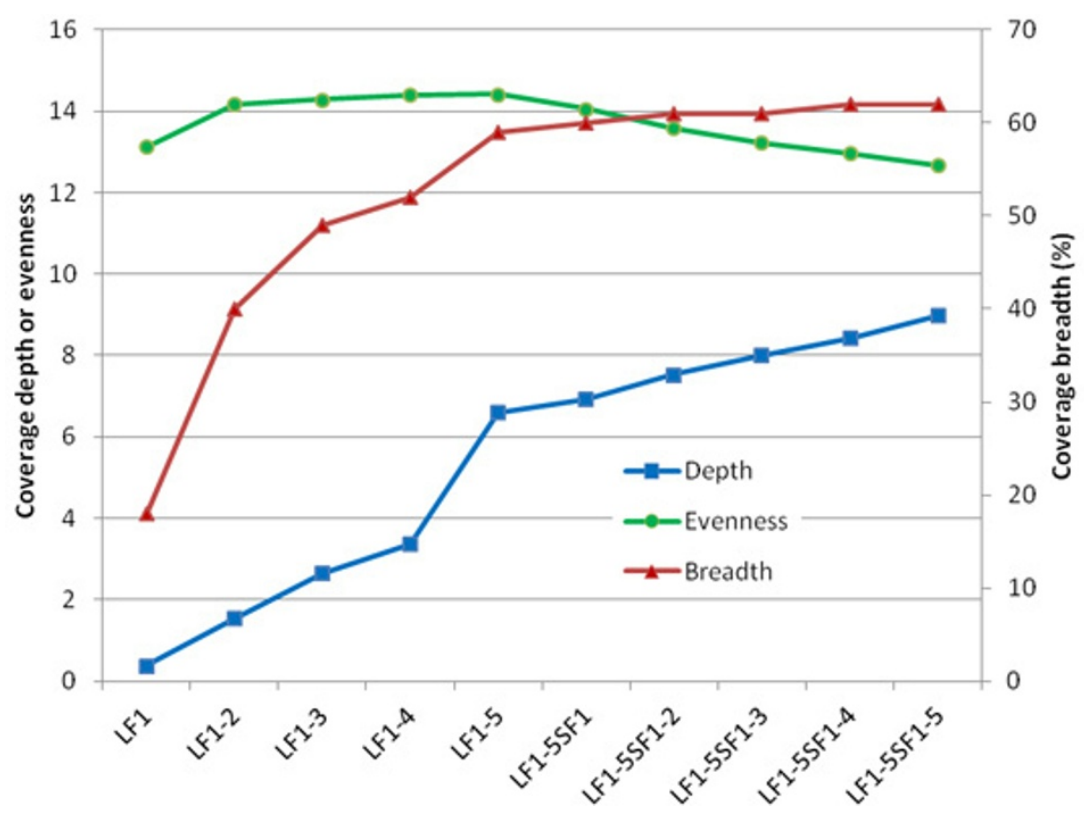

Figure 3 Change in genome coverage breadth, depth and evenness as more sequencing runs for the same TCO library were pooled and used as the input of SA_Run2Ref. See Table 2 for the sequencing runs pooled to form reads collections. 
(a) LF1
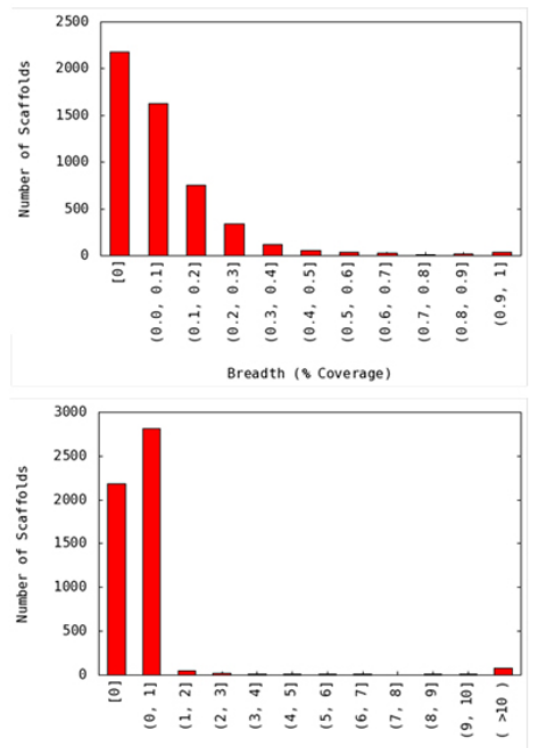

Depth (b) LF1-5

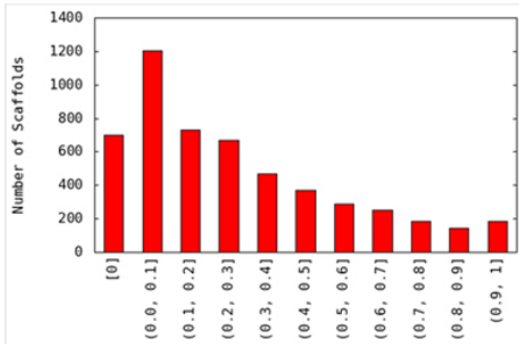

Breadth (\$ Coverage)

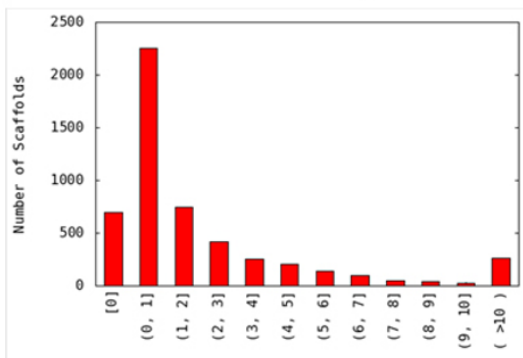

Depth (c) LF1-5SF1-5
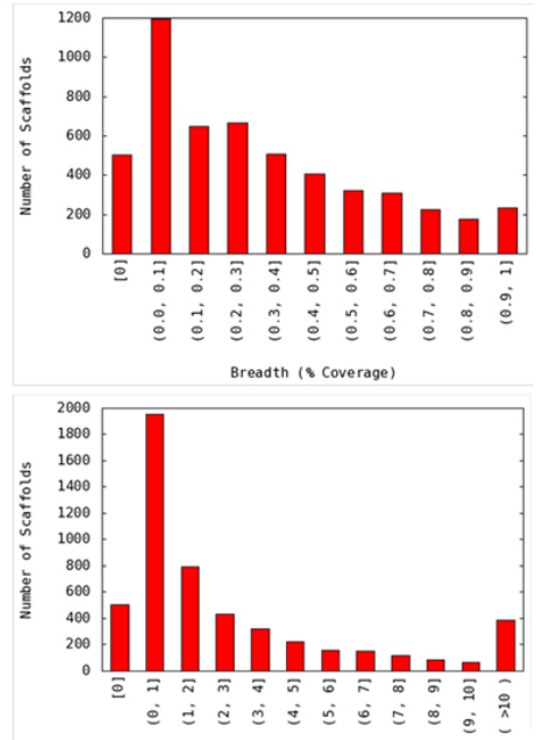

Depth

Figure 4 Change in the distribution of scaffold coverage breadth and depth as more sequencing runs for the same TCO library were pooled and used as the input of SA_Run2Ref. Shown are distributions for three reads collections: (a) LF1, (b) LF1-5, and (c) LF1-5SF1-5. See Table 2 for the sequencing runs pooled to form reads collections. Breadth and depth bins are open at the lower end and closed at the higher end, and breadth is expressed as percentage. For instance, $(0.3,0.4]$ stands for $30 \%<$ breadth $\leq 40 \%$, and $(0,1]$ stands for $0<$ depth $\leq 1$.

Table 3 The output statistics and derived statistics from running five pairs of NGS datasets through the SA_Run2Run workflow

\begin{tabular}{|c|c|c|c|c|c|c|c|c|c|c|}
\hline \multirow[t]{2}{*}{ NGS datasets } & \multicolumn{2}{|c|}{ Ecoli_I4M_R1 } & \multicolumn{2}{|c|}{ Ecoli_14M_R2 } & \multicolumn{2}{|c|}{ Ecoli_454_500K } & \multirow{2}{*}{$\begin{array}{c}\text { ECT_R1 } \\
\text { Run1 }\end{array}$} & \multirow{2}{*}{$\frac{\text { ECT_rerun_R1 }}{\text { Run2 }}$} & \multirow{2}{*}{$\begin{array}{c}\text { ECT_R2 } \\
\text { Run1 }\end{array}$} & \multirow{2}{*}{$\frac{\text { ECT_rerun_R2 }}{\text { Run2 }}$} \\
\hline & Run1 & Run2 & Run1 & Run2 & Run1 & Run2 & & & & \\
\hline \multicolumn{11}{|l|}{ Output statistics } \\
\hline $\begin{array}{l}\text { Total number of raw reads in } \\
\text { the run }\end{array}$ & $2,000,000$ & $2,000,000$ & $2,000,000$ & $2,000,000$ & 250,000 & 250,000 & $7,575,822$ & $7,064,035$ & $7,575,822$ & $7,064,035$ \\
\hline $\begin{array}{l}\text { Total number of cleaned reads } \\
\text { in the run }\end{array}$ & $1,968,732$ & $1,997,550$ & $1,999,692$ & $1,999,283$ & 231,123 & 231,245 & $7,538,930$ & $7,046,481$ & $7,542,743$ & $7,046,396$ \\
\hline $\begin{array}{l}\text { Number of unique reads in the } \\
\text { run (after removing identical } \\
\text { redundant reads) }\end{array}$ & $1,487,552$ & $1,482,834$ & $1,450,704$ & $1,405,779$ & 224,217 & 224,537 & $7,114,791$ & $6,702,601$ & $7,242,351$ & $6,839,442$ \\
\hline $\begin{array}{l}\text { Number of unique reads in the } \\
\text { run (after removing identical \& } \\
\text { inclusive redundant reads) }\end{array}$ & $1,487,552$ & $1,482,834$ & $1,450,704$ & $1,405,779$ & 221,379 & 221,622 & $6,885,175$ & $6,407,251$ & $6,945,743$ & $6,546,440$ \\
\hline $\begin{array}{l}\text { Total number of overlapping } \\
\text { reads in the run }\end{array}$ & 712,022 & 730,780 & 810,225 & 834,999 & 18,304 & 18,321 & 950,696 & 941,648 & 807,790 & 770,963 \\
\hline $\begin{array}{l}\text { Number of unique overlapping } \\
\text { reads in the run }\end{array}$ & 360,898 & 360,898 & 403,927 & 403,927 & 15,786 & 15,786 & 621,978 & 617,458 & 537,199 & 530,419 \\
\hline $\begin{array}{l}\text { Number of unique overlapping } \\
\text { reads from both runs }\end{array}$ & \multicolumn{2}{|c|}{360,898} & \multicolumn{2}{|c|}{403,927} & \multicolumn{2}{|c|}{15,786} & \multicolumn{2}{|r|}{625,044} & \multicolumn{2}{|r|}{538,561} \\
\hline \multicolumn{11}{|l|}{ Derived satistics } \\
\hline File size after preprocessing & $266 \mathrm{MB}$ & $266 \mathrm{MB}$ & $266 \mathrm{MB}$ & $266 \mathrm{MB}$ & $216 \mathrm{MB}$ & $216 \mathrm{MB}$ & $2.6 \mathrm{~GB}$ & $2.4 \mathrm{~GB}$ & $2.5 \mathrm{~GB}$ & $2.4 \mathrm{~GB}$ \\
\hline $\begin{array}{l}\text { Number of redundant cleaned } \\
\text { reads in the run }\end{array}$ & 481,180 & 514,716 & 548,988 & 593,504 & 9,744 & 9,623 & 653,755 & 639,230 & 597,000 & 499,956 \\
\hline $\begin{array}{l}\text { Redundancy rate within the } \\
\text { run }\end{array}$ & $32.3 \%$ & $34.7 \%$ & $37.8 \%$ & $42.2 \%$ & $4.4 \%$ & $4.3 \%$ & $9.5 \%$ & $10.0 \%$ & $8.6 \%$ & $7.6 \%$ \\
\hline $\begin{array}{l}\text { Total number of non- } \\
\text { overlapping reads in the run }\end{array}$ & $1,256,710$ & $1,266,770$ & $1,189,467$ & $1,164,284$ & 212,819 & 212,924 & $6,588,234$ & $6,104,833$ & $6,734,953$ & $6,275,433$ \\
\hline Number of unique non- & $1,126,654$ & $1,121,936$ & $1,046,777$ & $1,001,852$ & 205,593 & 205,836 & $6,263,197$ & $5,789,793$ & $6,408,544$ & $6,016,021$ \\
\hline
\end{tabular}


Table 3 The output statistics and derived statistics from running five pairs of NGS datasets through the SA_Run2Run workflow (Continued)

\begin{tabular}{|c|c|c|c|c|c|c|c|c|c|c|c|c|}
\hline $\begin{array}{l}\text { Number of redundant non- } \\
\text { overlapping reads in the run }\end{array}$ & 130,056 & 144,834 & 142,690 & 162,432 & 7,226 & 7,088 & 325,037 & & 315,040 & 326,409 & & 259,412 \\
\hline $\begin{array}{l}\text { Redundancy of non- } \\
\text { overlapping reads in the run }\end{array}$ & $11.5 \%$ & $12.9 \%$ & $13.6 \%$ & $16.2 \%$ & $3.5 \%$ & $3.4 \%$ & $5.2 \%$ & & $5.4 \%$ & $5.1 \%$ & & $4.3 \%$ \\
\hline $\begin{array}{l}\text { Number of redundant } \\
\text { overlapping reads in the run }\end{array}$ & 351,124 & 369,882 & 406,298 & 431,072 & 2,518 & 2,535 & 328,718 & & 324,190 & 270,591 & & 240,544 \\
\hline $\begin{array}{l}\text { Redundancy of overlapping } \\
\text { reads in the run }\end{array}$ & $97.3 \%$ & $102.5 \%$ & $100.6 \%$ & $106.7 \%$ & $16.0 \%$ & $16.1 \%$ & $52.9 \%$ & & $52.5 \%$ & $50.4 \%$ & & $45.3 \%$ \\
\hline $\begin{array}{l}\text { Total overlapping reads/total } \\
\text { cleaned reads (each run) }\end{array}$ & $36.2 \%$ & $36.6 \%$ & $40.5 \%$ & $41.8 \%$ & $7.9 \%$ & $7.9 \%$ & $12.6 \%$ & & $13.4 \%$ & $10.7 \%$ & & $10.9 \%$ \\
\hline $\begin{array}{l}\text { Total overlapping reads/total } \\
\text { cleaned reads (both runs) }\end{array}$ & \multicolumn{2}{|c|}{$36.4 \%$} & \multicolumn{2}{|c|}{$41.1 \%$} & \multicolumn{2}{|c|}{$7.9 \%$} & \multicolumn{3}{|c|}{$13.0 \%$} & \multicolumn{3}{|c|}{$10.8 \%$} \\
\hline Total runtime (min) & \multicolumn{2}{|c|}{13.3} & \multicolumn{2}{|c|}{13.0} & \multicolumn{2}{|c|}{8.2} & \multicolumn{3}{|c|}{106.3} & \multicolumn{3}{|c|}{110.8} \\
\hline
\end{tabular}

Overlapping reads are defined as those found in both runs. Unique overlapping reads from both runs are those left after removing redundant reads (identical or inclusive) from the combined overlapping reads from both runs.

redundancy rate than the 454 dataset, which is supported by not only the redundancy within the run $(32 \%$ to $42 \%$ vs. $4 \%$ ), but also the redundancy of overlapping reads between two split "runs" (97\% to $107 \%$ vs. $16 \%)$ (Table 3). For D. pulex genome re-sequencing, the two paired-end datasets had a relatively low redundancy level within each run ( $8 \%$ to $10 \%)$ and a low ratio of overlapping reads to total cleaned reads (11\% to $13 \%)$ (Table 3 and Figure 5). However, based on the output statistics from SA_Run2Ref, the rerun of the ECT library did not improve genome coverage breadth (Table 1 and Figure 2). It can be concluded from output information taken from both workflows that, despite nearly $90 \%$ of the reads from the two sequencing runs being different, they essentially cover the same scaffolds. If the main goal of the re-sequencing experiment was to obtain reads representing the entire genome, the additional run of the ECT library was apparently unnecessary.

With regard to redundant reads, a distinction is drawn between identical and inclusive reads. If reads are of the same length, there is no difference between these two types of redundant reads. Consequently, the number of unique overlapping reads in Run1 is the same as those in Run2 and both runs (see output statistics for Ecoli_I4M_R1 and Ecoli_I4M_R2 in Table 3 for examples). Preprocessing of Illumina datasets may cause length differentiation between cleaned reads. For datasets of reads with variable length, the number of unique reads after removing identical reads is higher than that after removing both types of redundant reads (see output statistics for Ecoli_454_500K, ECT and ECT_rerun datasets in Table 3 and Figure 5). As a result, the number of unique overlapping reads in Run1 may
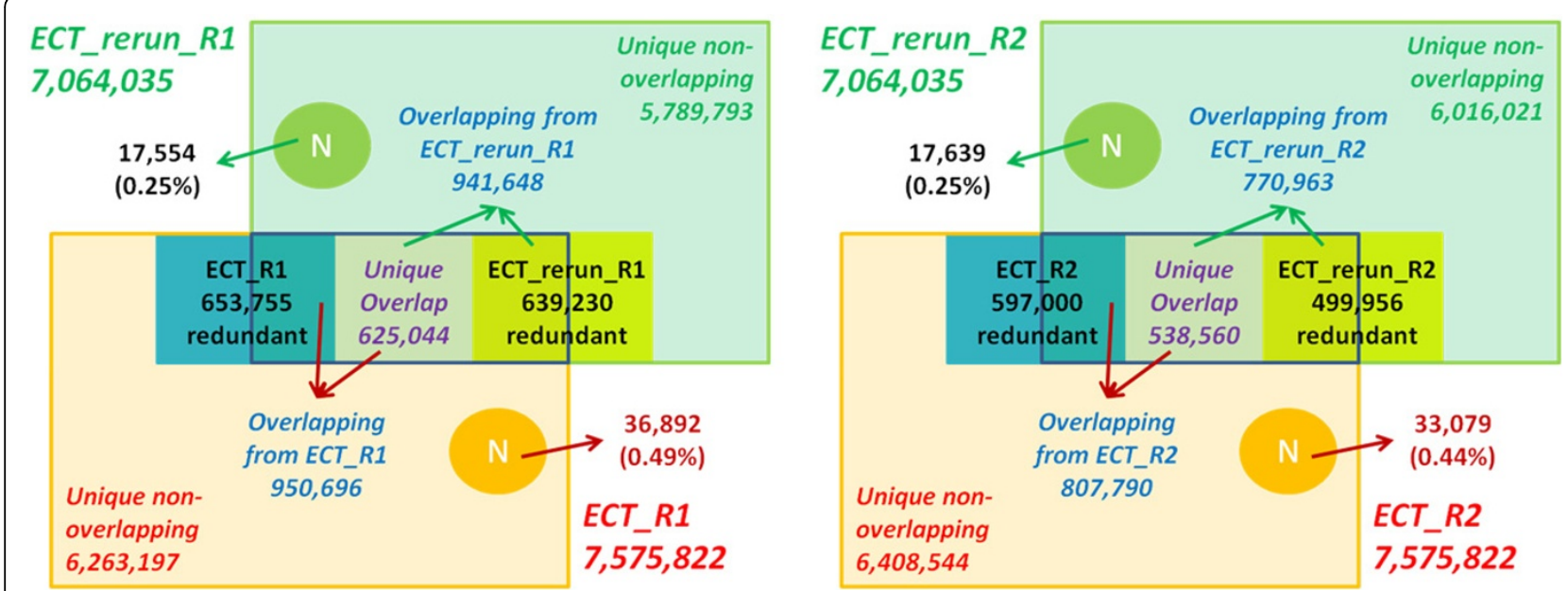

Figure 5 Breakdown of cleaned reads from two sequencing runs (ECT and ECT_rerun) into overlapping and non-overlapping reads based on the output from SA_Run2Run (see Table 3 for more info). " $N$ " represents reads containing $N$ that were removed during preprocessing. 
differ from that in Run2 and in both runs (see output statistics for ECT and ECT_rerun datasets in Table 3 and Figure 5).

\section{Analysis of the human genome re-sequencing dataset} Two paired-end sequencing runs, ERR032971 and ERR032972, within the ERX012406 dataset (NA12878) were analyzed using the three workflows to show the scalability of SeqAssist. Both runtime and memory usage were recorded and shown in Table 4 and Figure 6, respectively. The human genome is 15 times bigger than the $D$. pulex genome, and the size of the human genome re-sequencing runs in base pairs $(6.4 \sim 6.6 \mathrm{Gbp})$ is nearly triple that of $D$. pulex (2.1 2.2 Gbp, Table 1). However, the runtime of the human data through the SA_Run2Ref workflow (229 319 min, Table 4) was ca. 5.2 7.6 times that of the ECT $(44.6 \mathrm{~min})$ or the ECT_rerun $(42 \mathrm{~min})$ data (Table 1). For the SA_Run2Run workflow, their runtimes differed only by 3.5 -fold, i.e., $96 \sim 110 \mathrm{~min}$ for D. pulex vs. 348 381 min for human. The memory space consumed by SeqAssist when running the two human datasets did not exceed 10\% of the 284-GB RAM, except for a surge that occurred when multiple threads were used for calculating the statistics after the completion of BWA alignment at ca. 9700 seconds in the SA_Run2Ref workflow (Figure 6). These results suggest that SeqAssist is fully capable of handling any sequencing run data generated by current NGS platforms for organisms with a reference genome of any size and complexity, and results can be produced rapidly within a working day (less than 8 hours). This feature satisfies the demand for a quick turnaround from mega data to preliminary results.

\section{Conclusions}

We have demonstrated the main features of SeqAssist using multiple genome re-sequencing datasets. Output statistics from SeqAssist can guide the user in evaluating the quality of a DNA library prepared for genome re-

\begin{tabular}{|c|c|c|c|c|}
\hline Run data file & $\begin{array}{l}\text { Bases } \\
\text { (Gbp) }\end{array}$ & $\begin{array}{c}\text { SA_RunStats } \\
\text { (min) }\end{array}$ & $\begin{array}{c}\text { SA_Run2Ref } \\
\text { (min) }\end{array}$ & $\begin{array}{c}\text { SA_Run2Rur } \\
\text { (min) }\end{array}$ \\
\hline ERR032971_R1 & 3.2 & 194 & NA & 348 \\
\hline ERR032972_R1 & 3.3 & 208 & NA & \\
\hline ERR032971_R2 & 3.2 & 198 & NA & 381 \\
\hline ERR032972_R2 & 3.3 & 208 & NA & \\
\hline ERR032971 & 6.4 & NA & 229 & NA \\
\hline ERR032972 & 6.6 & NA & 319 & \\
\hline
\end{tabular}

The read length was 101 bases and the number of reads was $31.9 \mathrm{M}$ and 32.6 $M$ for ERR032971 and ERR032972, respectively. The runtime is rounded up to the closest minute. NA: not applicable. sequencing and in deciding whether there is a need to perform additional sequencing runs on the library. Based on the low coverage breadth (66\%) and the high reads mapping rate $(88 \%)$ (Table 1$)$, it appears that the ECT gDNA library may not be a good representation of the entire genome. The same holds true for the TCO library if considering its maximal breadth (62\%, Figure 3$)$ and mapping rate (84\%, Table 2 ). In terms of the number of multiplexed sequencing runs required for the TCO library, five runs of the large fraction seemed to be sufficient because they reached the maximal coverage breadth, with a total raw reads number of 4.2 million.

In summary, the SA_RunStats workflow generates basic statistics about an NGS dataset, including numbers of raw, cleaned, redundant and unique reads, redundancy rate, and a list of unique sequences with length and read count. The SA_Run2Ref workflow estimates the breadth, depth and evenness of genome-wide coverage of the NGS dataset at a nucleotide resolution. The SA_Run2Run workflow compares two NGS datasets to determine the redundancy (overlapping rate) between the two NGS runs. Statistics produced by SeqAssist or derived from SeqAssist output files are designed to inform the user: whether, what percentage, how many times and how evenly a genomic locus (i.e., gene, scaffold, chromosome or genome) is covered by sequencing reads, how redundant the sequencing reads are in a single run or between two runs.

SeqAssist is a useful and informative tool that can serve as a valuable "assistant" to a broad range of investigators who conduct genome re-sequencing, RNA-Seq, or de novo genome sequencing and assembly experiments. For RNASeq experiments, SeqAssist output files that contain unique sequences along with their mapped genomic loci and copy numbers may be readily transformed into gene expression data. An investigator who de novo assembles a genome from sequencing data may use SeqAssist to map the original reads to the assembled genome and obtain a ratio of mapped to cleaned reads. As an additional parameter to existing metrics [18], this ratio can be used to objectively compare the quality of different assemblies made from the same sequencing data. For further indepth analyses of NGS data, one is advised to use other appropriate tools available from the bioinformatics community. For instance, one may choose to apply spliced aligners such as RUM [19] and SpliceSeq [20] to identify splice junctions for alternative splicing detection of RNASeq data, or employ a structural variant (SV) discovery software such as BreakDancer [21], Pindel [22] and PRISM [23] to call SV events and discern breakpoints from genome re-sequencing data.

We plan to improve visualization features of SeqAssist in the future versions. Specifically, the nucleotideresolution mapping and coverage depth (copy number) information generated from SA_Run2Ref shall be 

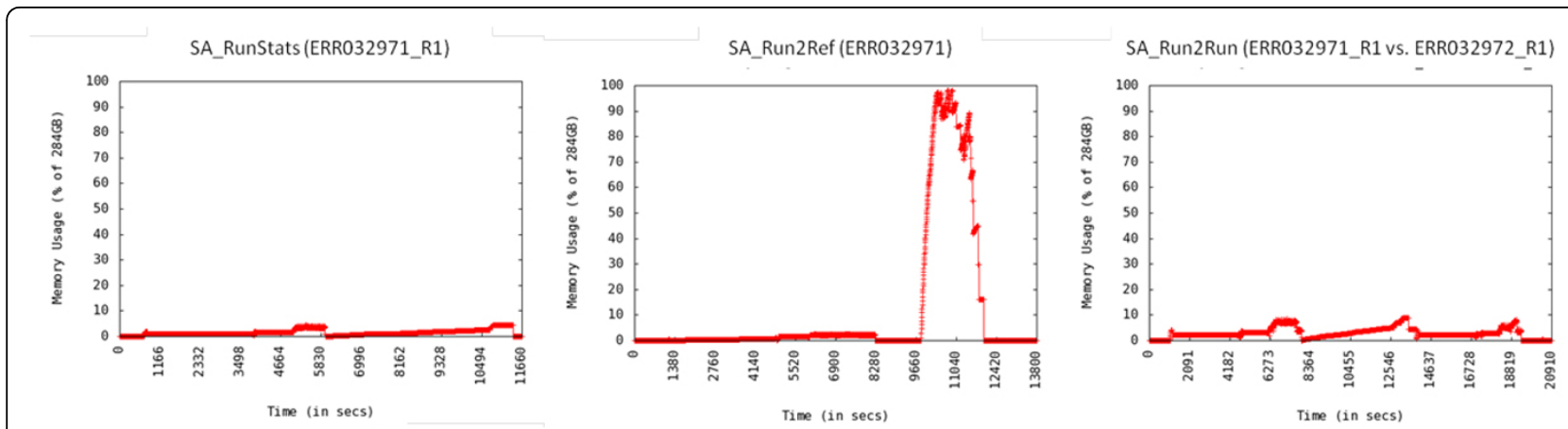

Figure 6 Memory usage recorded every 5 seconds when running the human genome re-sequencing data through the three SeqAssist workflows.

transformed into interactive visual graphics to allow the user to visualize gene coverage or expression levels.

\section{Availability and requirements}

- Project name: SeqAssist

- Project home page: http://orca.st.usm.edu/cbbl/ seqassist/

- Operating systems: Linux (Ubuntu)

- Programming language: Perl v.5.14.2 with the following packages: Parallel::ForkManager and Getopt::Long

- Other requirements: BWA (http://bio-bwa.sourceforge.net/), Cutadapt (https://code.google.com/p/ cutadapt/), GNUplot, and Java Runtime Environment (JRE version 1.6.0_30 or greater)

- License: Free for commercial and academic uses.

- Any restrictions to use by non-academics: None.

\section{Competing interests}

The authors declare that they have no competing interests.

\section{Authors' contributions}

PG and CZ conceived the project. PG designed the software architecture. YP implemented the code. NW and PG supervised the coding. ASM developed the GUI. ASM and YP conducted the computational experiments. CZ, NW and PG supervised the experiments. NDB, JGL, AJK and PG generated the Daphnia pulex sequencing data. PG, ASM and YP drafted the manuscript. All authors revised, read and approved the manuscript.

\section{Acknowledgements}

This work was supported by the U.S. Army Environmental Quality and Installation Technology Basic Research Program and the National Science Foundation under award number EPS 0903787. Permission to publish this information was granted by the Chief of Engineers.

\section{Declarations}

Publication charges for this article were funded by the U.S. Army Environmental Quality and Installation Technology Basic Research Program. This article has been published as part of BMC Bioinformatics Volume 15 Supplement 11, 2014: Proceedings of the 11th Annual MCBIOS Conference. The full contents of the supplement are available online at http://www. biomedcentral.com/bmcbioinformatics/supplements/15/S11.

\section{Authors' details}

'School of Computing, University of Southern Mississippi, Hattiesburg, MS 39406, USA. ${ }^{2}$ Badger Technical Services, LLC, San Antonio, TX 78216, USA.
${ }^{3}$ Environmental Laboratory, U.S. Army Engineer Research and Development Center, Vicksburg, MS 39180, USA.

Published: 21 October 2014

\section{References}

1. Mardis ER: Next-generation DNA sequencing methods. Annu Rev Genomics Hum Genet 2008, 9:387-402.

2. Mardis ER: Next-generation sequencing platforms. Annu Rev Anal Chem (Palo Alto Calif) 2013, 6:287-303.

3. Martin JA, Wang Z: Next-generation transcriptome assembly. Nat Rev Genet 2011, 12:671-682.

4. Wang Z, Gerstein M, Snyder M: RNA-Seq: a revolutionary tool for transcriptomics. Nat Rev Genet 2009, 10:57-63.

5. Bernardi G, Wiley EO, Mansour H, Miller MR, Orti G, Haussler D: The fishes of Genome 10K. Mar Genomics 2012, 7:3-6.

6. Genome 10K: a proposal to obtain whole-genome sequence for 10,000 vertebrate species. J Hered 2009, 100:659-674, Genome 10K Community of Scientists.

7. Wong PB, Wiley EO, Johnson WE, Ryder OA, O'Brien SJ, Haussler D, et al: Tissue sampling methods and standards for vertebrate genomics. Gigascience 2012, 1:8.

8. The i5K Initiative: advancing arthropod genomics for knowledge, human health, agriculture, and the environment. J Hered 2013, 104:595-600, i5K Consortium.

9. Levine R: i5k: The 5,000 Insect Genome Project. Am Entomol 2011, 72:110-113.

10. Stratton M: Genome resequencing and genetic variation. Nat Biotechnol 2008, 26:65-66.

11. Abecasis GR, Auton A, Brooks LD, DePristo MA, Durbin RM, Handsaker RE, et al: An integrated map of genetic variation from 1,092 human genomes. Nature 2012, 491:56-65.

12. Stephens PJ, Tarpey PS, Davies H, Van LP, Greenman C, Wedge DC, et al: The landscape of cancer genes and mutational processes in breast cancer. Nature 2012, 486:400-404.

13. Li H, Durbin R: Fast and accurate short read alignment with BurrowsWheeler transform. Bioinformatics 2009, 25:1754-1760.

14. Li H, Durbin R: Fast and accurate long-read alignment with BurrowsWheeler transform. Bioinformatics 2010, 26:589-595.

15. Li H, Handsaker B, Wysoker A, Fennell T, Ruan J, Homer N, et al: The Sequence Alignment/Map format and SAMtools. Bioinformatics 2009, 25:2078-2079.

16. Blattner FR, Plunkett G, Bloch CA, Perna NT, Burland V, Riley M, et al: The complete genome sequence of Escherichia coli K-12. Science 1997, 277:1453-1462.

17. Colbourne JK, Pfrender ME, Gilbert D, Thomas WK, Tucker A, Oakley TH, et al: The ecoresponsive genome of Daphnia pulex. Science 2011, 331:555-561.

18. Bradnam KR, Fass JN, Alexandrov A, Baranay P, Bechner M, Birol I, et al: Assemblathon 2: evaluating de novo methods of genome assembly in three vertebrate species. Gigascience 2013, 2:10. 
19. Grant GR, Farkas MH, Pizarro AD, Lahens NF, Schug J, Brunk BP, et al: Comparative analysis of RNA-Seq alignment algorithms and the RNASeq unified mapper (RUM). Bioinformatics 2011, 27:2518-2528.

20. Ryan MC, Cleland J, Kim R, Wong WC, Weinstein JN: SpliceSeq: a resource for analysis and visualization of RNA-Seq data on alternative splicing and its functional impacts. Bioinformatics 2012, 28:2385-2387.

21. Chen K, Wallis JW, McLellan MD, Larson DE, Kalicki JM, Pohl CS, et al: BreakDancer: an algorithm for high-resolution mapping of genomic structural variation. Nat Methods 2009, 6:677-681.

22. Ye K, Schulz MH, Long $Q$, Apweiler $R$, Ning Z: Pindel: a pattern growth approach to detect break points of large deletions and medium sized insertions from paired-end short reads. Bioinformatics 2009, 25:2865-2871.

23. Jiang $Y$, Wang $Y$, Brudno M: PRISM:pair-read informed split-read mapping for base-pair level detection of insertion, deletion and structural variants. Bioinformatics 2012, 28:2576-2583.

doi:10.1186/1471-2105-15-S11-S10

Cite this article as: Peng et al:: SeqAssist: a novel toolkit for preliminary analysis of next-generation sequencing data. BMC Bioinformatics 2014 15(Suppl 11):S10.

\section{Submit your next manuscript to BioMed Central} and take full advantage of:

- Convenient online submission

- Thorough peer review

- No space constraints or color figure charges

- Immediate publication on acceptance

- Inclusion in PubMed, CAS, Scopus and Google Scholar

- Research which is freely available for redistribution

Submit your manuscript at www.biomedcentral.com/submit 